\title{
Deformation Feature of Goss Grains in Fe-3\%Si Steel Focused on Stored Energy after Cold Rolling
}

\author{
Hyung-Ki Park ${ }^{1}$, Se-Jong Kim ${ }^{1}$, Heung Nam Han ${ }^{1}$, Chan-Hee Han ${ }^{2}$ and Nong-Moon Hwang ${ }^{1, *}$ \\ ${ }^{1}$ Department of Materials Science and Engineering, Seoul National University, Seoul 151-742, Korea \\ ${ }^{2}$ POSCO Technical Research Laboratories, POSCO, Pohang 790-360, Korea
}

\begin{abstract}
In an effort to explain the selective Goss abnormal grain growth in Fe-3\%Si steel based on the sub-boundary enhanced solid-state wetting, we investigated why sub-boundaries are formed exclusively in Goss grains after primary recrystallization. In order for Goss grains to have subboundaries exclusively, they should undergo only recovery without recrystallization whereas other grains undergo recrystallization. For this, Goss grains should have the lowest stored energy after cold rolling. Goss and rotated cube orientations have the same and the lowest Taylor factor among the grains formed after cold rolling, implying that they should have the lowest stored energy. The stored energy between Goss and rotated cube orientations was compared by the crystal plasticity finite element calculation, which showed that the stored energy of the Goss orientation was lower than that of the rotated cube orientation after plane strain deformation. [doi:10.2320/matertrans.M2010029]
\end{abstract}

(Received January 25, 2010; Accepted June 24, 2010; Published August 11, 2010)

Keywords: cold rolling, Taylor factor, crystal plasticity finite element method (CP-FEM), electron backscattering diffraction (EBSD)

\section{Introduction}

Grain-oriented $\mathrm{Fe}-3 \% \mathrm{Si}$ steel is a soft magnetic material that is widely used as a core material of pole and power transformers. The low core loss can be achieved by the highly textured microstructure with Goss orientation, $\{110\}\langle 001\rangle$ because $\langle 001\rangle$ direction in iron is the easiest magnetization direction with low magnetic losses. ${ }^{1)}$ Such a strong Goss texture could be obtained after secondary recrystallization which is also referred to as abnormal grain growth (AGG). However, the mechanism of selective Goss AGG has not yet been clearly understood since its first report by Goss in 1935. ${ }^{2)}$

As to the origin of Goss grains, Inokuti et $a l .{ }^{3)}$ made an extensive study and suggested that abnormally-growing Goss grains during secondary recrystallization should be inherited from the Goss grains formed in the subsurface layer by shear stress during hot rolling. ${ }^{4-7)}$ According to this suggestion, the subsurface layer containing Goss grains should play an important role in inducing AGG. This aspect was confirmed by the observation that when the subsurface layer containing the Goss texture was removed, AGG occurred incompletely during secondary recrystallization. ${ }^{8,9)}$ Therefore, the inheritance of Goss grains throughout the cold rolling seems to be closely related with the mechanism of Goss-selective AGG in the secondary recrystallization of $\mathrm{Fe}-3 \%$ Si steel.

To explain the selective AGG of Goss grains in Fe-3\% Si steel, Hwang et al. ${ }^{10-12)}$ suggested a mechanism of subboundary enhanced solid-state wetting. According to this mechanism, the grains with sub-boundaries have a high probability to grow by solid-state wetting and thereby can grow abnormally. If the Goss grains have exclusively subboundaries of very low energy after primary recrystallization, the selective Goss AGG can be explained. Ushigami et al. ${ }^{13)}$ observed sub-boundaries in the abnormally-growing Goss

*Corresponding author, E-mail: nmhwang@snu.ac.kr grains by in-situ synchrotron X-ray during secondary recrystallization of $\mathrm{Fe}-3 \% \mathrm{Si}$ steel. Also, Dorner et al. ${ }^{14)}$ confirmed the existence of sub-boundaries exclusively within Goss grains after primary recrystallization of a sheet sample reduced $89 \%$ by cold rolling of a single crystal of Goss orientation.

An additional effort was made by Park et al. ${ }^{15)}$ to confirm whether the existence of sub-boundaries is a unique feature of abnormally-growing Goss grains. According to this observation, indeed the existence of sub-boundaries is exclusive in abnormally-growing Goss grains. The microstructure of abnormally-growing Goss grains shows typically sub-boundaries and stepped dislocation networks, which are the microstructural feature of recovery. For example, Fig. 1(a) and 1(b) show a sub-boundary and stepped dislocation networks, respectively, in the initial stage of secondary recrystallization observed by annular dark field scanning transmission electron microscope. The distance between the individual dislocations in Fig. 1(a) was $123.33 \mathrm{~nm}$ and from this the boundary angle of the sub-boundary was estimated to be $0.115^{\circ}$ (Refer to Ref. 15) for details about calculation of boundary angle). They are a low energy dislocation structure (LEDS), which is expected to have been formed by recovery during primary recrystallization. Such LEDSs were observed only in abnormally-growing Goss grains.

In order for Goss grains to have LEDSs exclusively after primary recrystallization, they should undergo only recovery without recrystallization, which implies that Goss grains should have the lowest stored energy among grains of various orientations formed after cold rolling. The purpose of this study is to examine such a possibility using crystal plasticity finite element method (CP-FEM) under a plane strain condition.

\section{Experimental Procedure}

An industrial hot-rolled sample of $\mathrm{Fe}-3 \% \mathrm{Si}$, which employed AlN as an inhibitor of grain growth to produce 
(a)

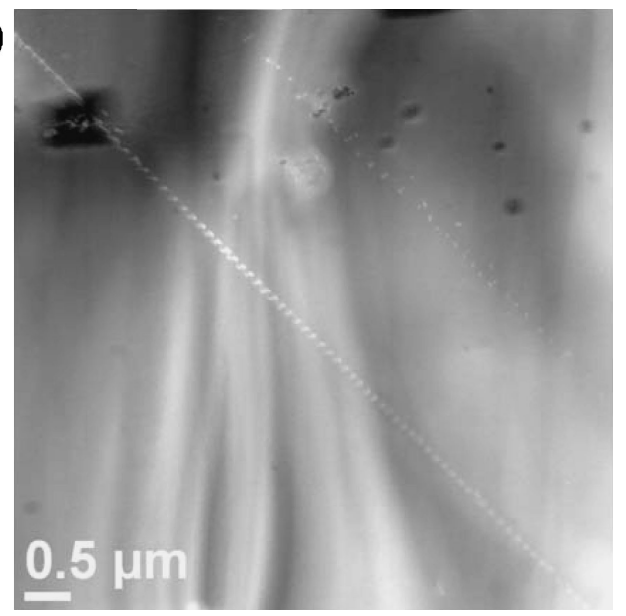

(b)

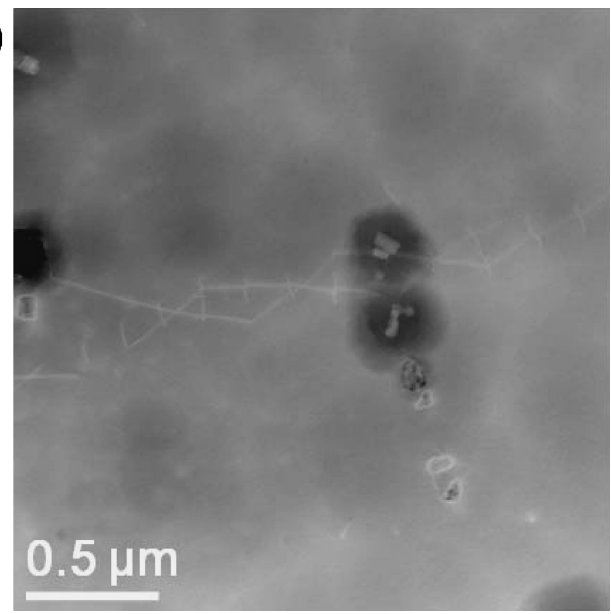

Fig. 1 (a) Sub-boundary and (b) stepped-dislocation networks in the initial stage of secondary recrystallization observed by annular dark field scanning transmission electron microscope.

the highly grain-oriented (HGO) electrical steel, was used as a starting material to investigate the retention of Goss grains during cold-rolling. The hot-rolled sample with a thickness of $2.3 \mathrm{~mm}$ was cold-rolled by a laboratory rolling mill to $0.3 \mathrm{~mm}$, which is equivalent to a total engineering thickness reduction of $\varepsilon=87 \%$. Orientations of the hot-rolled and cold-rolled samples were investigated by electron backscattered diffraction (EBSD), attached to a field emission scanning electron microscopy (FE-SEM) (JEOL JSM 6500F). The software (EDAX/TSL) was used for the analysis of orientation data.

\section{Simulation Procedure}

The deformation behavior of a bicrystal was calculated using a crystal plasticity model based on a rate-dependent slip system constitutive relation. In the calculation, we used the crystal kinematics defined by Asaro ${ }^{16)}$ and the implicit time integration procedure of the constitutive equation suggested by Kalidindi et al. ${ }^{17)}$ The constitutive model and time-integration scheme have been implemented in ABAQUS ${ }^{18)}$ using an user material subroutine (UMAT). The shear rates were given in terms of the resolved shear stress on the active slip systems and the resistance of the active slip systems. The shear rate on the slip system was described by the following equation. ${ }^{19)}$

$$
\dot{\gamma}^{\mathrm{s}}=\dot{\gamma}_{0}^{\mathrm{s}}\left|\frac{\tau^{\mathrm{s}}}{\tau_{0}^{\mathrm{s}}}\right|^{1 / m} \operatorname{sign}\left(\tau^{\mathrm{s}}\right),
$$

where $\dot{\gamma}_{0}^{\mathrm{s}}, \mathrm{m}, \tau^{\mathrm{s}}$, and $\tau_{0}^{\mathrm{s}}$ are the reference shearing rate, rate sensitivity of slip, resolved shear stress, and slip resistance, respectively. The slip resistance is expressed as

$$
\dot{\tau}_{0}^{\mathrm{s}}=\sum_{\beta} h^{\mathrm{s} \beta}\left|\dot{\gamma}^{\beta}\right|
$$

where $h^{\mathrm{s} \beta}$ is the rate of strain hardening on the slip system $s$ due to shearing on the slip system $\beta$. The hardening coefficients are

$$
h^{\mathrm{s} \beta}=h^{\beta}\left(q+(1-q) \delta_{\mathrm{s} \beta}\right), \quad h^{\beta}=h_{0}\left(1-\frac{\tau_{0}^{\mathrm{s}}}{g_{\mathrm{s}}}\right)^{a},
$$

Table 1 Material parameters for CP-FEM simulation.

\begin{tabular}{lccccccc}
\hline \multirow{2}{*}{ Elastic Moduli ${ }^{20)}$} & \multicolumn{3}{c}{$E$} & \multicolumn{3}{c}{$v$} \\
\cline { 2 - 7 } & \multicolumn{3}{c}{$211 \mathrm{GPa}$} & \multicolumn{2}{c}{0.29} \\
\hline \multirow{2}{*}{ Plastic parameters $^{17)}$} & $\dot{\gamma}$ & $m$ & $a$ & $h_{0}$ & $g_{0}$ & $q$ \\
\cline { 2 - 7 } & 0.001 & 0.07 & 2.25 & $180 \mathrm{MPa}$ & $148 \mathrm{MPa}$ & 1.0 \\
\hline
\end{tabular}

where $q$ determines latent-to-self hardening, $h_{0}$ is a reference self-hardening coefficient, $a$ is the hardening exponent, and $g_{\mathrm{s}}$ is the slip resistance at which the self and latent hardening coefficients asymptote to zero. The material properties are listed in Table $1 .{ }^{17,20)}$ The rate of plastic work per unit volume was calculated considering a work conjugate as in the following eq. ${ }^{21)}$

$$
\dot{w}=\left(C^{*} \sigma^{*}\right) L^{\mathrm{p}}
$$

where

$$
\begin{aligned}
& C^{*}=F^{\mathrm{e} T} F^{\mathrm{e}}, \quad \sigma^{*}=F^{\mathrm{e}-1}\{\operatorname{det}(F) \sigma\} F^{\mathrm{e}-T} \\
& \text { and } L^{\mathrm{P}}=\dot{F}^{\mathrm{p}} F^{\mathrm{p}-1}
\end{aligned}
$$

where $F^{\mathrm{e}}, F^{\mathrm{p}}$, and $\sigma$ represent elastic deformation gradient, plastic deformation gradient, and Cauchy stress, respectively.

Figure 2 shows the initial condition of the finite element mesh. An aggregate of 4096 crystals is represented by $64 \times$ 64 two-dimensional four-node finite elements (CPE4). ${ }^{18)}$ Each element represents one crystal. The initial configuration of mesh is designated by 'center' and 'surround'. The center region is placed at the bottom in the left side of Fig. 2, and consists of $20 \times 20$ elements. The simulations were performed under a plane strain condition. Arrows in Fig. 2 represent the compressive direction. To represent the symmetry, we introduced the symmetry lines along normal direction (ND) and rolling direction (RD), respectively, at the left and the bottom as circles in Fig. 2, so that the displacement is constrained along RD in the left and along ND in the bottom. The displacement along both ND and RD is constrained at the intersection of both symmetry lines, which is represented by a triangle in Fig. 2. Displacement boundary conditions for plane strain compression were applied to surface nodes. Due to the symmetric condition 


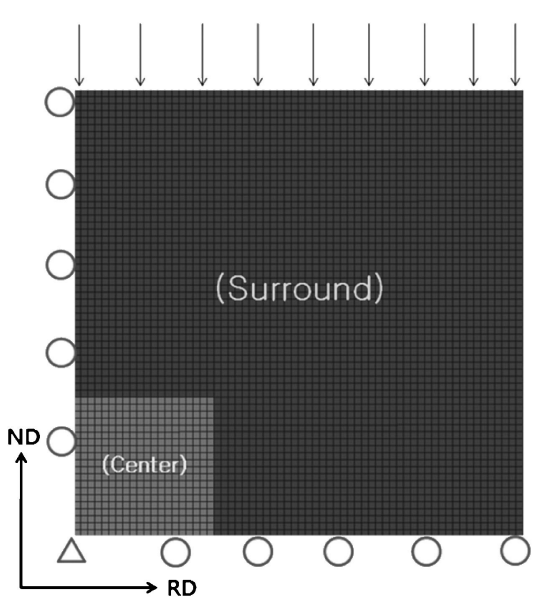

Fig. 2 The initial finite element mesh with the bicrystal mesh constituted by $64 \times 64$ two-dimensional four-node finite elements $(\mathrm{CPE} 4)$.

of the mesh, a quarter of system with a square-shaped grain at the center enclosed by a surrounding square-shaped grain was simulated. The plane strain deformation was calculated up to $30 \%$ reduction. 48 slip systems of bcc, which consist of $12 \times\{110\}\langle 111\rangle, 12 \times\{112\}\langle 111\rangle$, and $24 \times\{123\}\langle 111\rangle$, were considered in the finite element method (FEM) calculations. The percentage of orientation keeping its initial orientation was analyzed and the accumulated plastic strain energy for each element was calculated by eq. (4) at every step.

\section{Results}

The subsurface layer containing Goss grains was investigated by EBSD at each cold rolling stage to examine the retention of the Goss grains during cold rolling. Figure 3(a), (b), (c), and (d) show inverse pole figure maps for ND-RD sections after $0 \%, 35 \%, 57 \%$, and $87 \%$ cold rolling, respectively. The orientation of green colored grains in the circle was a Goss orientation, $\{110\}\langle 001\rangle$. As the thickness was reduced by cold rolling, the size of remaining Goss grains decreased noticeably. However, Goss grains with very small size tended to survive near the subsurface layer even after $87 \%$ reduction (Fig. 3(d)) in spite of the fact that the Goss orientation is unstable under plane strain deformation. These survived Goss grains are expected to undergo only recovery without recrystallization and, as a result, to produce sub-boundaries, which are LEDS, exclusively after primary recrystallization. In order for the Goss grains to undergo recovery exclusively, their stored energy should be the lowest among the grains formed after cold rolling. In order to confirm this, the stored energy of Goss grains after deformation should be compared with that of grains with other orientations.

Mishra et $a{ }^{.7)}$ analyzed the textures formed after hot and cold rolling in the $\mathrm{Fe}-3 \% \mathrm{Si}$ steel using X-ray. After hot rolling, the $\{011\}\langle 100\rangle$ texture was developed at the surface whereas the $\{112\}\langle 110\rangle$ and $\{001\}\langle 110\rangle$ textures were developed at the center. These textures were deformed and rotated during cold rolling, as a result of which (a)
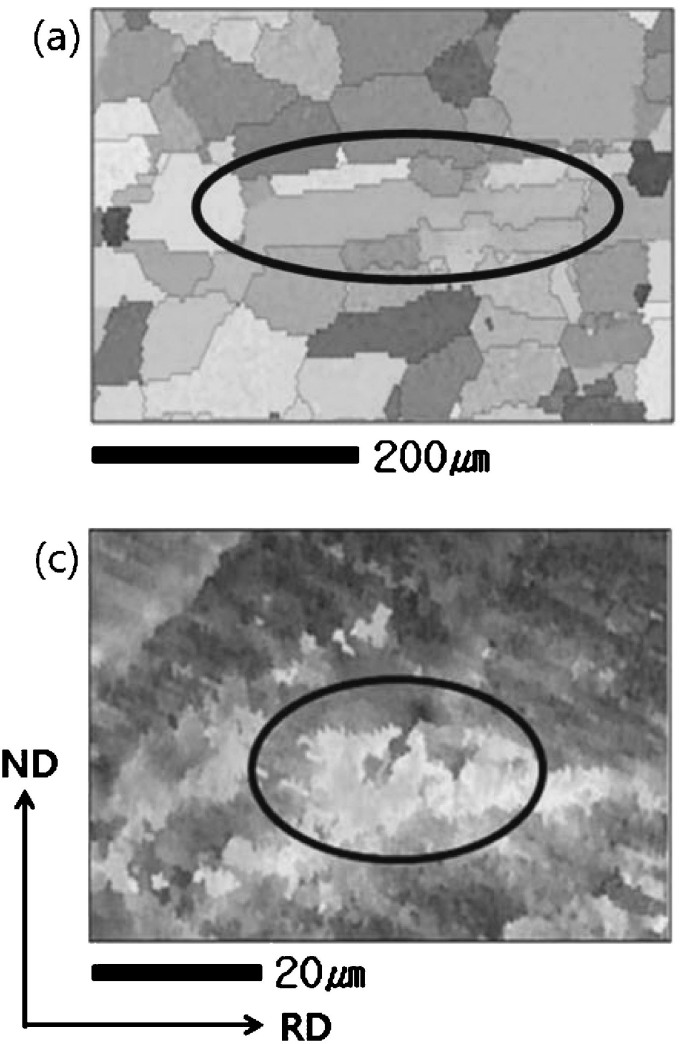

(b)

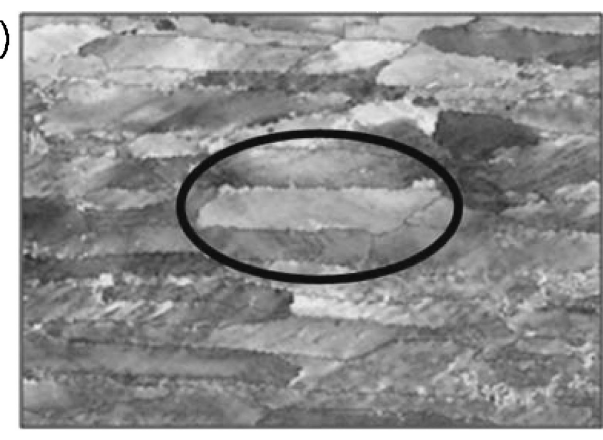

$100 \mu \mathrm{m}$

(d)

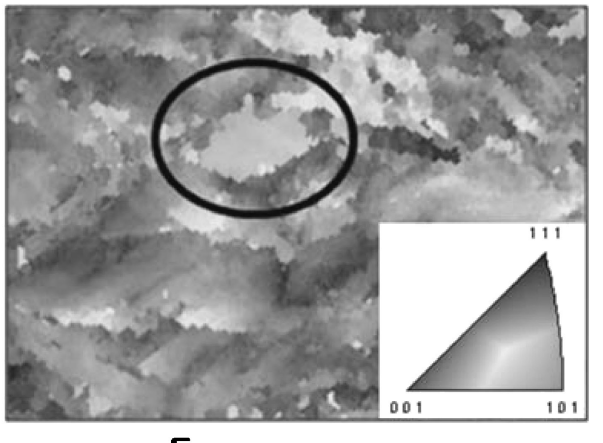

$5 \mu \mathrm{m}$

Fig. 3 Inverse pole figure maps of a subsurface layer obtained by EBSD for the ND-RD section after hot rolling and after cold rolling with thickness reductions of (a) $0 \%$, (b) $35 \%$, (c) $57 \%$, and (d) $87 \%$. The green colored grains in the circle have a Goss orientation of $\{110\}\langle 001\rangle$. 

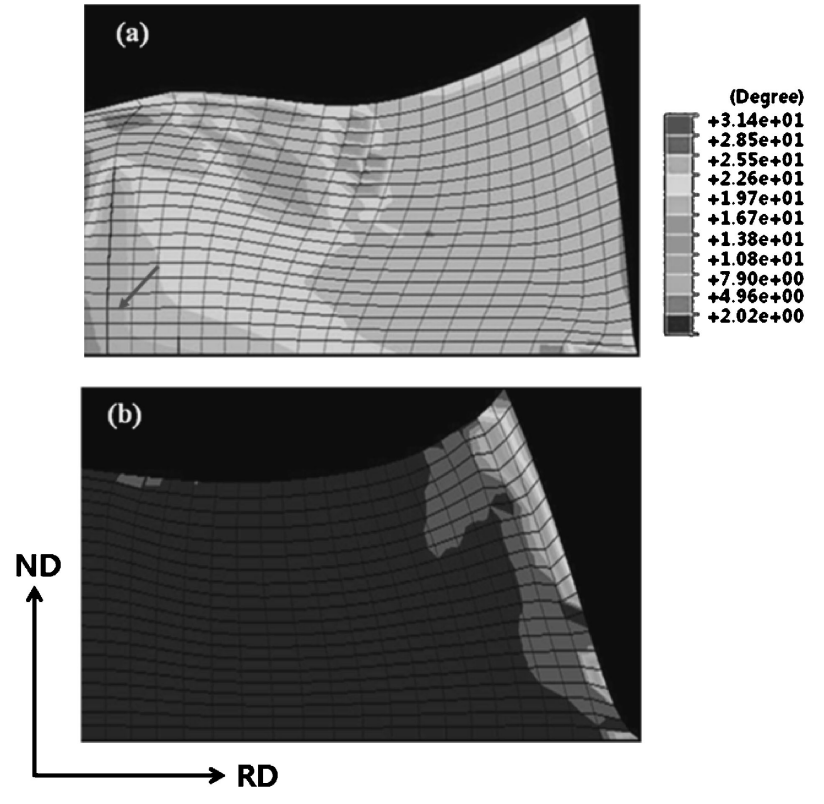

Fig. 4 Deformed meshes of ND-RD section in the center of (a) Goss and (b) rotated cube orientations after $30 \%$ reduction. The rotation from the initial orientation increases as the color changes from the blue to the red.

major textures of $\{001\}\langle 110\rangle,\{112\}\langle 110\rangle,\{111\}\langle 112\rangle$, and $\{111\}\langle 110\rangle$ as well as a minor texture of $\{011\}\langle 100\rangle$ were developed. Among these orientations Goss, $\{110\}\langle 001\rangle$, and rotated cube, $\{001\}\langle 110\rangle$, orientations have the same Taylor factor which is lower than other orientations. ${ }^{20)}$ This is why we focused on the Taylor factor of Goss and rotated cube orientations although orientations rotated from Goss and rotated cube by 10 degree along a transverse direction have the lowest Taylor factor in all orientations. The stored energy of grains depends on the Taylor factor under the same amount of deformation. Therefore, they are expected to have lower stored energy than other orientations. It remains to determine which has the lower stored energy between Goss and rotated cube orientations. The stored energy depends not only on the Taylor factor but also on other factors such as crystal lattice rotation and work hardening behavior. Since CP-FEM calculation considers all such factors, it is known to determine the most reliable stored energy. In order to compare the stored energy between Goss and rotated cube orientations, CP-FEM simulations were carried out to calculate the plastic strain energy during deformation.

The simulations were carried out for two conditions. One is that the Goss orientation was located in the center surrounded by $\{111\}\langle 112\rangle$ orientation, which is observed dominantly in the cold-rolled sample. The other is that the rotated cube orientation was located in the center surrounded by $\{111\}\langle 112\rangle$ orientation in order to compare the stored energy between Goss and rotated cube orientations under the same condition of neighboring grains because the deformation of grains depends not only on their intrinsic orientation but also on the orientation of neighboring grains. Figure 4(a) and 4(b) show the deformed meshes in the center, which were initially Goss and rotated cube orientations, after $30 \%$ reduction, respectively. The color scale

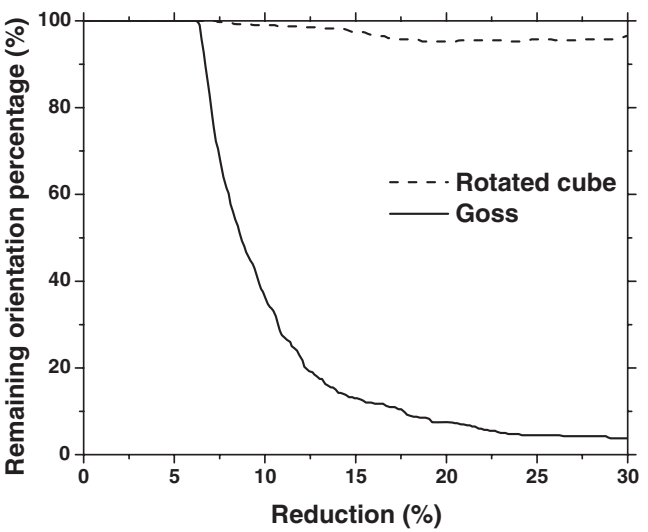

Fig. 5 Remaining percentages of Goss and rotated cube orientations in the center during plane strain deformation.

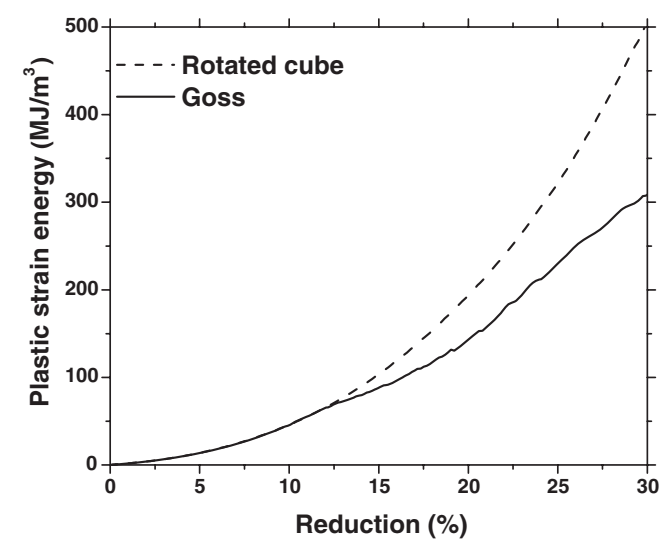

Fig. 6 The plastic strain energy for Goss and rotated cube orientations during plane strain deformation.

represents the angle rotated from the initial orientation. It is assumed that the orientations are not changed if they are deviated less than $10^{\circ}$ from the initial orientation. Most of Goss orientations were rotated from the initial orientation and only a few of them kept their orientation at the bottom in the left side as indicated by a red arrow in Fig. 4(a) whereas most of the rotated cube orientations survived after deformation.

Figure 5 shows the percentage of remaining orientations with deformation for Goss and rotated cube orientations. After about $6 \%$ plane strain compression, the Goss orientation started to rotate and as a result the percentage of the remaining Goss orientation decreased dramatically. Eventually, about $3.8 \%$ of the Goss orientation remained after $30 \%$ reduction. However, the rotated cube orientation was much more stable under plane strain compression than the Goss orientation. About $96 \%$ of the rotated cube orientations kept their orientation after $30 \%$ deformation.

Figure 6 shows the plastic strain energy for the reduction of Goss and rotated cube orientations. In both cases, the plastic strain energy tended to increase as the reduction increased. However, the increasing rate of the plastic strain energy for the rotated cube orientation was much faster than that for the Goss orientation. The plastic strain energy after $30 \%$ reduction of the Goss orientation was about 


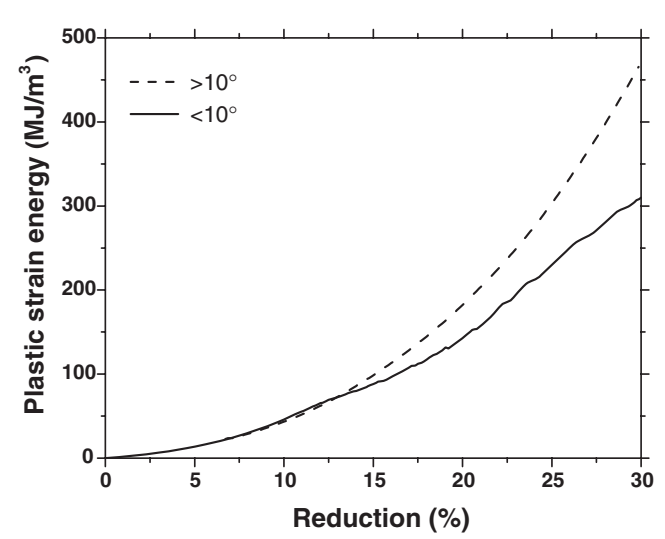

Fig. 7 The plastic strain energy with reduction for the orientations deviating less and larger than $10^{\circ}$ from the Goss orientation.

$40 \%$ lower than that of the rotated cube orientation. This result indicates that the remaining Goss orientation stored lower plastic energy during cold rolling than the remaining rotated cube orientation although they have the same Taylor factor.

Figure 7 shows the dependence of the plastic strain energy on the reduction for the orientations deviating less and larger than $10^{\circ}$ from the Goss orientation. The orientations deviating less than $10^{\circ}$ have lower plastic strain energy than those deviating larger than $10^{\circ}$. This result indicates that the remaining Goss orientation should have lower plastic strain energy than the orientations rotated from the Goss orientation.

\section{Discussion}

Although the Goss orientation is not stable under a plane strain deformation, Goss grains with very small size were tended to survive near the subsurface layer even after $87 \%$ reduction (Fig. 3(d)). This aspect of Goss survival was also reported by Inokuti et al. ${ }^{3)}$ and Dorner et $a .^{22)}$ CP-FEM results of plane strain deformation support the survival of Goss grains after cold rolling (Fig. 5). The Goss orientation which is unstable under a plane strain deformation was rotated during deformation; however, about $3.8 \%$ of the Goss orientation remained after $30 \%$ reduction. On the other hand, the rotated cube orientation which is stable under a plane strain deformation remained their orientation during deformation. This result agrees well not only with the well-known experimental observation of Goss survival as shown in Fig. 3 and but also with the previous report that the Goss orientation decreased dramatically as the plane strain compression increased $^{22)}$ and that the rotated cube is a stable orientation under the plane strain condition. ${ }^{23,24)}$

To examine the possibility of formation of sub-boundary exclusively in Goss grains, the plastic strain energy was compared between Goss and rotated cube orientations. These two orientations have the low and the same Taylor factor under a plane strain deformation and thereby are expected to have the same stored energy after deformation if only the Taylor factor is considered. However, the stored energy of Goss orientation turned out to be $40 \%$ lower than that of the rotated cube orientation after $30 \%$ reduction (Fig. 6).
Although these two orientations have the same Taylor factor, they have different characteristics in the plane strain deformation. This different characteristic seems to be related with the fact that the Goss orientation is unstable whereas the rotated cube orientation is stable. The unstable Goss orientation is more easily rotated than the stable rotated cube orientation. Therefore, the remaining Goss grain might be less deformed because its rotated part accommodates the applied stress.

During the plane strain deformation most of Goss orientations were rotated to two symmetrical $\{111\}\langle 112\rangle$ orientations but a small percentage of Goss orientations remained with deviation less than $10^{\circ}$. The stored energy of these two parts, rotated and remaining Goss orientations, was compared in Fig. 7. Under a plane strain deformation the Taylor factor of Goss orientation is lower than that of $\{111\}\langle 112\rangle$ orientation. ${ }^{14)}$ Therefore, the orientations deviating less than $10^{\circ}$ from the Goss orientation underwent the deformation with a low Taylor factor, whereas the orientations deviating larger than $10^{\circ}$ underwent the deformation with a high Taylor factor. As a result, the remaining Goss orientation would store lower plastic strain energy.

\section{Conclusions}

The plastic strain energy was compared by CP-FEM between Goss and rotated cube orientations, which have the same and the lowest Taylor factor among the grains after cold rolling. The Goss orientation had lower plastic strain energy than the rotated cube orientation. This result supports the possibility that the Goss orientation should have the lowest stored energy among the grains after cold rolling and undergo only recovery during primary recrystallization, producing sub-boundaries whereas grains of other orientations undergo recrystallization, producing general boundaries.

\section{Acknowledgements}

This work was financially supported by POSCO Technical Research Laboratories and the National Research Foundation of Korea (NRF) grant funded by the Korea government (MEST) (No. M10600000159-06J0000-15910). Heung Nam Han and Se-Jong Kim were supported by the National Research Foundation of Korea (NRF) grant funded by the Korea government (MEST) (2009-0083038).

\section{REFERENCES}

1) H. Honda and S. Kaya: Sci. Rep. Tohoku Imp. Univ. 15 (1926) 721.

2) N. P. Goss: Trans. Am. Soc. Met. 23 (1935) 511.

3) Y. Inokuti, C. Maeda and Y. Ito: ISIJ Int. 70 (1984) 2057.

4) M. Matsuo, T. Sakai and S. Yozo: Metall. Trans. 17A (1986) 1313.

5) M. Matsuo: ISIJ Int. 29 (1989) 809.

6) Y. Shimizu, Y. Ito and Y. Iida: Metall. Trans. 17A (1986) 1323.

7) S. Mishra, C. Därmann and K. Lüke: Acta Metall. 32 (1984) 2185.

8) A. Böttcher and K. Lücke: Acta Metall. Mater. 41 (1993) 2503.

9) N. C. Pease, D. W. Jones, M. H. L. Wise and W. B. Hutchinson: Met. Sci. 15 (1981) 203.

10) K. J. Ko, P. R. Cha, D. Srolovitz and N. M. Hwang: Acta Mater. 57 (2009) 838.

11) D. K. Lee, K. J. Ko, B. J. Lee and N. M. Hwang: Scr. Mater. 58 (2008) 683. 
12) D. K. Lee, B. J. Lee, K. J. Ko and N. M. Hwang: Mater. Trans. 50 (2009) 2521-2525.

13) Y. Ushigami, Y. Suga, N. Takahashi, K. Kawasaki, Y. Chikaura and H Kii: J. Mater. Eng. 13 (1991) 113-118.

14) D. Dorner, L. Lahn and S. Zaefferer: Mater. Sci. Forum 467 (2004) 129

15) H. K. Park, S. D. Kim, S. C. Park, J. T. Park and N. M. Hwang: Scr. Mater. 62 (2010) 376.

16) R. J. Asaro: Adv. Appl. Mech. 23 (1983) 1.

17) S. R. Kalidindi, C. A. Bronkhorst and L. Anand: J. Mech. Phys. Solids 40 (1922) 537
18) ABAQUS (version 6.5) Analysis User's manual, (Hibbitt, Karlsson, and Sorensen Inc., Pawtucket, 2004).

19) D. Peirce, R. J. Asaro and A. Needelman: Acta Mater. 33 (1985) 923.

20) D. N. Lee: Texture and related phenomena, (The Korean Institute of Metals and Materials, Seoul, 2006).

21) L. Anand: Int. J. Plust. 1 (1985) 213.

22) D. Dorner, S. Zaefferer and D. Raabe: Acta Mater. 55 (2007) 2519

23) J. Boeslau and D. Raabe: Mater. Sci. Forum 157-162 (1994) 501.

24) D. Raabe: Steel Res. 66 (1995) 222. 